\title{
Auslandstätigkeiten und das Recht auf polnische Überbrückungsrenten
}

\author{
Marcin Zieleniecki
}

A. Die historische Entwicklung des Rechts auf vorzeitige Pensionierung infolge besonderer Arbeitsbedingungen

B. Reformbestrebungen

C. Die Einführung der Überbrückungsrente

D. Die Berücksichtigung von Auslandstätigkeiten

E. Die Etablierung eines Systems zum elektronischen Datenaustausch

A. Die historische Entwicklung des Rechts auf vorzeitige Pensionierung infolge besonderer Arbeitsbedingungen

Seit Mitte der fünfziger Jahre des 20. Jahrhunderts können unter besonderen Bedingungen beschäftigte Arbeitnehmer das im polnischen Sozialversicherungssystem verankerte Recht auf Pensionierung vor Erreichen des allgemein vorgeschriebenen Pensionierungsalters nutzen. Voraussetzung dafür ist, dass die ausgeführte Tätigkeit in einem beträchtlichen Grad gesundheitsschädlich oder beschwerlich ist oder hohe psychischkörperliche Ansprüche stellt. Dem trägt die Verordnung vom 25. Juni 1954 über die allgemeine Altersversorgung von Arbeitnehmern und deren Familien ${ }^{1}$ Rechnung, die bei zur so genannten Kategorie I gehörenden Arbeitnehmern die Berechtigung auf Altersrente für Frauen im Alter von 55 Jahren und für Männer im Alter von 60 Jahren vorsieht. Jedoch setzt dies voraus, dass der gesamte Beschäftigungszeitraum 20 Jahre (für Frauen) bzw. 25 Jahre (für Männer) beträgt und davon zumindest 15 Jahre unter Bedingungen der Kategorie I gearbeitet wurde. Begleitet wurde dies von der Anwendung günstigerer Berechnungsgrundsätze im Bereich der Altersversorgung, der Invalidenrente und der Gewährung von Zuschüssen. Ähnliche Modelle wurden im genannten Zeit-

1 GBl. der Republik Polen Nr. 30, Pos. 116. 
raum in allen damaligen sozialistischen Ländern und in den achtziger Jahren des 20. Jahrhunderts auch in einigen westlichen Ländern eingeführt. ${ }^{2}$

In den folgenden Jahren wurde das Recht auf vorzeitige Altersversorgung infolge besonderer Arbeitsbedingungen beträchtlich ausgedehnt. 1956 erfolgte eine Ausweitung der zu einer Frühpensionierung berechtigenden Tätigkeiten von 100 auf 1643. In den sechziger Jahren umfasste der Katalog der unter diese Kategorie fallenden Arbeiten bereits 339 und 1975 waren es über 500 Tätigkeitsbereiche ${ }^{4}$. Obwohl diese Zahl durch die Verordnung des Ministerrates vom 4. Mai $1979^{5}$ auf 278 gesenkt wurde, waren 1983 unter besonderen Bedingungen Beschäftigte in 344 Bereichen in 14 Wirtschaftszweigen sowie auch Beschäftigte in sieben Tätigkeitsarten mit besonderem Charakter mit dem Recht auf Frühpensionierung ausgestattet. ${ }^{6}$ Nach Einschätzung von $W$. Muszalski stieg infolge der Änderungen des Sozialversicherungsgesetzes in der ersten Hälfte der achtziger Jahre die Zahl der unter besonderen Bedingungen eingestellten Beschäftigten auf 1.817.000 und im Geltungsbereich des Altersversorgungsgesetzes aus dem Jahr $1982^{7}$ nutzte die Mehrheit der Beschäftigten die Möglichkeit einer früheren Pensionierung ${ }^{8}$. Ein niedrigeres Rentenalter wurde zur Regel statt zur Ausnahme. Diese Situation hielt bis zum Ende der neunziger Jahre des 20. Jahrhunderts an. Im Jahr 1998 betrug das Durchschnittsrentenalter in Polen 58,7 Jahre bei Männern und 54,7 Jahre bei Frauen. ${ }^{9}$ Angesichts des ab Mitte der neunziger Jahre auch in Polen zu verzeichnenden Anstiegs der Lebensdauer hatte dies einen beträchtlichen Einfluss auf die finanzielle Lage des Sozialversicherungssystems.

2 Muszalski, Zatrudnienie a ubezpieczenie społeczne (Beschäftigung und Sozialversicherung), Warschau 1992, S. 134.

3 Verordnung des Ministerrates vom 10. September 1956 über die Zugehörigkeit von Arbeitnehmern zu Beschäftigungskategorien (GBl. der Republik Polen Nr. 39, Pos. 176).

4 Verordnung des Ministerrates zur Änderung der Verordnung über die Zugehörigkeit von Arbeitnehmern zu Beschäftigungskategorien: 1) vom 26. August 1964 (GBl. der Republik Polen Nr. 34, Pos. 215), 2) vom 29. Juni 1967 (GBl. der Republik Polen Nr. 26, Pos. 121), 3) vom 27. Juni 1968 (GBl. der Republik Polen Nr. 22, Pos. 148), 4) vom 23. Mai 1975 (GBl. der Republik Polen Nr. 18, Pos. 96).

5 Verordnung des Ministerrates vom 4. Mai 1979 über die erste Beschäftigungskategorie (GBl. der Republik Polen Nr. 13, Pos. 86).

6 Verordnung des Ministerrates vom 7. Februar 1983 über das Pensionierungsalter von in besonderen Bedingungen oder mit besonderem Charakter tätigen Arbeitnehmer (GBl. der Republik Polen Nr. 8, Pos. 43).

7 Gesetz vom 14. Dezember 1982 über die Altersversorgung von Arbeitnehmern und deren Familien (GBl. der Republik Polen Nr. 40, Pos. 267).

8 Muszalski, Zatrudnienie a ubezpieczenie społeczne (Fußn. 2), S. 135-136.

9 Stachura, Wcześniejsze emerytury a emerytury pomostowe (Beschäftigung und Sozialversicherung), Przegląd Ubezpieczeń Społecznych i Gospodarczych (Überblick Sozial- und Wirtschaftsversicherungen) Nr. 8/2000, S. 43. 


\section{B. Reformbestrebungen}

Eines der grundlegenden Ziele der in Polen am 1. Januar 1999 in Angriff genommenen Rentenreform war die schrittweise Abschaffung der Möglichkeit einer Pensionierung vor Erreichung der vorgeschriebenen Altersgrenze. Das Gesetz über die Pensionen und Renten aus dem FUS (staatlicher Garantiefonds für Sozialversicherungsleistungen) ${ }^{10}$ bestätigte die bisherige Möglichkeit einer früheren Pensionierung ausschließlich für vor dem 1. Januar 1949 geborene Versicherte sowie für Versicherte der Jahrgänge 1949 bis 1968, die innerhalb von zehn Jahren ab Beginn der Rentenreform (d. h. bis 31. Dezember 2008) besondere Anforderungen erfüllten. Die übrigen Versicherten hatten somit kein Recht mehr auf eine Altersversorgung vor Erreichen des gesetzlichen Rentenalters. Eine Ausnahme war in Art. 24 Pkt. 2 und 3 des Rentengesetzes vorgesehen. Hier wurde ein gesondertes Gesetz für Überbrückungsrenten für unter besonderen Bedingungen Beschäftigte oder für Tätigkeiten mit besonderem Beschäftigungscharakter angekündigt.

\section{Die Einführung der Überbrückungsrente}

Die Idee der Überbrückungsrente wurde aber erst am 19. Dezember 2008 realisiert, als das Gesetz über Überbrückungsrenten vom Parlament verabschiedet wurde ${ }^{11}$. In Kraft trat dieses Gesetz mit dem 1. Januar 2009.

Dieses Gesetz begrenzte das Spektrum besonderer Berechtigungen auf vorzeitige Altersversorgung maßgeblich. Umgesetzt wurde dies zum einen durch die Einführung neuer Kataloge von Tätigkeiten, die zur Überbrückungsaltersversorgung berechtigen, zum anderen durch den nun erlöschenden Charakter dieser Leistung.

Die Bestimmung von Tätigkeitsarten unter besonderen Bedingungen sowie mit besonderem Charakter erfolgte unter Mitarbeit von Experten aus der Arbeitsmedizin sowie von gesellschaftlichen Partnern, die die dreiseitige Kommission für soziale und wirtschaftliche Angelegenheiten repräsentieren. Ergebnis war die Ausarbeitung von 1. neuen Definitionen für Tätigkeiten unter besonderen Bedingungen und mit besonderem Charakter, 2. von einem Katalog von Risikofaktoren in besonderen Arbeitsumfeldbedingungen und 3. von einem Katalog von Tätigkeiten unter besonderen Bedingungen und mit besonderem Charakter ${ }^{12}$.

10 Gesetz vom 17. Dezember 1998 über Pensionen und Renten aus dem FUS (GBl. der Republik Polen Nr. 39, Pos. 353).

11 Gesetz vom 19. Dezember 2008 über Überbrückungsrenten (GBl. der Republik Polen Nr. 237, Pos. 1656).

12 Zieleniecki, Uwagi na temat charakteru prawnego emerytury pomostowej (Bemerkungen zum rechtlichen Charakter der Überbrückungsrente), Z zagadnie zabezpieczenia społecznego (Zu Fragen der 
Die in Art. 1 Pkt. 1 des Gesetzes über die Überbrückungsrenten enthaltene Definition für Tätigkeiten unter besonderen Bedingungen umfasst zum einen Tätigkeiten mit Risikofaktoren, die mit dem Alter mit großer Wahrscheinlichkeit eine dauerhafte Gesundheitsschädigung verursachen könnten, zum anderen solche Tätigkeiten, die unter besonderen, von Kräften der Natur oder technologischen Prozessen determinierten Arbeitsumfeldbedingungen ausgeführt werden, die den Arbeitnehmer trotz des Einsatzes von technischen, organisatorischen und medizinischen Vorsorgemaßnahmen überfordern, so dass im Ergebnis eine Alterung noch vor Erreichen des Rentenalters erfolgt und eine Tätigkeit auf dem bisherigen Arbeitsplatz erschwert ist. Als besondere, von Naturkräften bestimmte Arbeitsbedingungen sind Arbeiten unter Tage, auf dem Wasser, unter Wasser und in der Luft anzusehen. Die besonderen, durch technologische Vorgänge bestimmten Arbeitsbedingungen sind Tätigkeiten in heißem oder kaltem Mikroklima oder in erhöhtem Luftdruck sowie Tätigkeiten verbunden mit großer körperlicher Anstrengung und hoher Belastung durch eine erzwungene unveränderbare Körperhaltung.

Die in Art. 3 Abs. 1 des Gesetzes über Überbrückungsrenten enthaltene Definition verknüpft den Begriff der besonderen Tätigkeit mit besonderen Risikofaktoren und einer besonderen geistig-körperlichen Beanspruchung bei deren Ausführung sowie mit der Minderung der Leistungsfähigkeit, um die Gesundheit und das Leben der Arbeitnehmer als Ausprägung der öffentlichen Sicherheit infolge einer Verschlechterung der geistig-körperlichen Leistungsfähigkeit aus Altersgründen nicht zu gefährden ${ }^{13}$.

Die durch den Gesetzgeber vorgegebenen Definitionen von Tätigkeiten unter besonderen Bedingungen und Tätigkeiten mit besonderem Charakter sind im Grunde ohne rechtliche Bedeutung: für die Bewertung des Rechts auf Überbrückungsaltersversorgung ist nämlich allein der Katalog dieser Tätigkeiten maßgeblich. ${ }^{14}$ Beide Definitionen spielten somit eine lediglich unterstützende Rolle bei der Ausarbeitung der Anlagen Nr. 1 und 2 zum genannten Gesetz. In dem Katalog wurde das Recht auf vorzeitige Altersversorgung 40 Tätigkeiten unter besonderen Bedingungen und 24 Tätigkeiten mit besonderem Charakter zuerkannt. Diese Einschränkung der Berechtigung auf vorzeitige Einstellung der Berufsausübung findet ihre Begründung im technischen bzw. technologischen Fortschritt, im verbesserten Arbeitsschutz und in der medizinischen Entwicklung, wodurch die seit 1983 bestehenden Kataloge der zu einer vorzeitigen Altersversorgung berechtigenden Tätigkeiten an Aktualität verloren. ${ }^{15}$

Sozialversorgung), Zeszyty Naukowe Uniwersytetu Gdańskiego (Wissenschaftliche Hefte der Universität Gdansk) Nr. 2 (2010), S. 120.

13 Zieleniecki, Emerytura pomostowa w nowym systemie emerytalnym (Überbrückungsrente im neuen Rentensystem), Fundacja Rozwoju Uniwersytetu Gdańskiego (Stiftung für Entwicklung der Universität Gdansk), Danzig 2011, S. 228.

14 Florek, Opinia Rady Legislacyjnej z 28 lipca 2008 o projekcie ustawy o emeryturach pomostowych RL 0303-54/08 (Gutachten des Legislativen Rates vom 28. Juli 2008 zum Entwurf des Gesetzes über Überbrückungs-Altersversorgung), Przegląd Legislacyjny (Legislative Übersicht) Nr. 3/2008, S. 211.

15 Zieleniecki, Emerytura pomostowa w nowym systemie emerytalnym (Fußn. 13), S. 229-230. 
Ausdruck findet der Übergangscharakter der Überbrückungsrente darin, dass sie ausschließlich solchen Arbeitnehmern zuerkannt wird, die ihre berufliche Tätigkeit unter besonderen Bedingungen vor dem 1. Januar 1999 aufgenommen haben. Der Gesetzgeber geht also davon aus, dass das Recht auf Überbrückungsaltersversorgung ausschließlich den Beschäftigten zusteht, die ihre qualifizierte Berufstätigkeit vor dem Inkrafttreten der Rentenreform aufgenommen haben. Diese Leistung dient somit in erster Linie einer Realisierung der Rentenanwartschaft von unter besonderen Bedingungen oder mit besonderem Charakter tätigen Arbeitnehmern, von denen vor 1999 ein Anstieg zu verzeichnen war. Dabei gewährleistet das Gesetz nicht die Verwirklichung aller Rentenanwartschaften dieser Arbeitnehmer. Art. 4 Pkt. 6 und Art. 49 Pkt. 3 des Gesetzes über die Überbrückungsrenten gestehen das Recht auf Überbrückungsrente ausschließlich einem engen Kreis von Personen zu, die qualifizierte Arbeiten nach den neuen strengeren Kriterien nach dem 31. Dezember 2008 ausüben. Das Recht auf diese Altersversorgung verloren damit alle Beschäftigten, die ihre Tätigkeit unter besonderen Bedingungen oder mit besonderem Charakter nach dem 31. Dezember 1998 aufgenommen hatten. Das Gesetz besagt, dass sich die Zahl der Arbeitnehmer auf dem Arbeitsmarkt, die die Voraussetzungen für die Berechtigung auf Überbrückungsrente erfüllen, durch die Einführung der neuen Definitionen von Tätigkeiten unter besonderen Bedingungen und mit besonderem Charakter weiter verringern wird, und zwar bis zum völligen Erlöschen des Rechts auf Überbrückungsrente bei Pensionierung aus dem FUS der letzten Gruppe von zur Überbrückungsrente berechtigten Arbeitnehmern ${ }^{16}$.

Voraussetzung für die Berechtigung auf Überbrückungsrente ist der Nachweis von 15 Arbeitsjahren ${ }^{17}$ unter besonderen Bedingungen oder mit besonderem Tätigkeitscharakter durch den Beschäftigten. Wie bereits erwähnt dient dieses Gesetz den Versicherten, die die nachzuweisenden Arbeitszeiträume sowohl vor dem 1. Januar 1999 als auch nach dem 31. Dezember 2008 erreicht haben. Die Festlegung der Arbeitszeiträume dafür erfolgt auf Grundlage zweier verschiedenartiger Rechtsformen - erstens nach den im Gesetz über Altersversorgung und Renten aus dem FUS und in der Verfügung des Ministerrates vom 7. Februar 1983 verankerten Vorschriften und zweitens nach den Vorschriften des Gesetzes über Überbrückungsrenten.

Die Beschäftigungszeit unter besonderen Bedingungen sowie mit besonderem Charakter in der Zeit vor dem 1. Januar 2009 wird auf der Grundlage schriftlicher vom Arbeitgeber ausgestellter und sich auf entsprechende Unterlagen gründender Bescheinigungen festgelegt. Diese Bescheinigungen haben Namen und Vornamen des Arbeitnehmers, Datum der Aufnahme und des Abschlusses der Beschäftigung sowie auch An-

16 Zieleniecki, Emerytura pomostowa w nowym systemie emerytalnym (Fußn. 13), S. 224-225.

17 Im Einklang mit Art. 8 Pkt. 2 und Art. 10 Pkt. 2 des Gesetzes über Überbrückungsrenten ist ein zehnjähriger Beschäftigungszeitraum unter besonderen Bedingungen oder mit besonderem Charakter erforderlich für Taucher und Caissoniere, Arbeiter in Hochdruckkammern, Hochseefischer, bei der Verarbeitung von Asbest oder bei Abbrucharbeiten von mit Asbest belasteten Gebäuden beschäftigte Arbeiter sowie Mitglieder von Berufsbergrettungsgruppen. 
gaben zur Beschäftigungsart und zum Arbeitsplatz in den einzelnen Zeitabschnitten zu enthalten. ${ }^{18}$ Die Abwicklung vieler Betriebe, unvollständige Unterlagen über die ausgeführten Beschäftigungen unter besonderen Bedingungen im Unternehmen sowie auch das Fehlen solcher Unterlagen in neu entstandenen Unternehmen erschwerten vielen Versicherten den Nachweis der sie zur Frühpensionierung berechtigenden Beschäftigungszeiträume. Die vor dem 1. Januar 2009 den Beschäftigungszeitraum unter besonderen Bedingungen oder mit besonderem Charakter bestätigende und vom Arbeitgeber ausgestellte Bescheinigung ist jedoch nicht der einzig zulässige Beweis. Ist es nicht möglich, dass der Versicherte eine entsprechende Bescheinigung vorlegt, hat das Rentenorgan andere Beweismittel wie Dienstausweise, Gewerkschaftsausweise, Arbeitsverträge, Einträge in Personalausweise sowie auch an den Arbeitnehmer während des Beschäftigungszeitraums gerichtete Schreiben anzuerkennen. ${ }^{19}$ Nachweis für die Ausführung von Tätigkeiten unter besonderen Bedingungen oder mit besonderem Charakter kann auch das vom Arbeitgeber ausgestellte Arbeitszeugnis sein. In der Literatur wird ein solches Arbeitszeugnis zur Kategorie der Bescheinigungen des Unternehmens im Sinne von $\S 21$ Abs. 2 der Verordnung über das Vorgehen bei Pensions- und Rentenleistungen und die Grundsätze der Auszahlung dieser Leistungen gezählt. ${ }^{20}$

Das Gesetz über Überbrückungsrenten führt eine laufende Erfassung der nach dem 31. Dezember 2008 erzielten Zeiträume einer Beschäftigung unter besonderen Bedingungen oder mit besonderem Charakter ein. Angaben zu den Zeiträumen, die zur Überbrückungsrente berechtigen, sind 1. die Erfassung der solche Arbeiten ausführenden Arbeitnehmer, 2. das Zentralregister der betreffenden Arbeitnehmer in der Staatlichen Sozialversicherungsanstalt (ZUS) und 3. das individuelle Konto des Versicherten bei der ZUS. Mit der Inbetriebnahme des Systems der laufenden Erfassung der Beschäftigungszeiträume unter besonderen Bedingungen oder mit besonderem Charakter durch die ZUS veränderte sich die rechtliche Lage des Versicherten bei der Nachweiserbringung von Beschäftigungszeiträumen nach Art. 3 Abs. 1 und 3 des Gesetzes über Überbrückungsaltersversorgung. Die Angaben zur Anerkennung von auf dem individuellen Konto sowie im Zentralregister erfassten Beschäftigungszeiträumen des Versicherten nach dem Jahr 2008 haben Tatsachencharakter, sind dem Rentenorgan von Amts wegen bekannt und erfordern keine Beweisführung. Art. 77 § 4 des Verwaltungsverfahrensgesetzbuches verlangt vom Rentenorgan einzig die Benachrichtigung der Parteien.

$18 \S 21$ Abs. 4 der Verordnung des Ministerrates vom 7. Februar 1983 über das Vorgehen bei Pensions- und Rentenleistungen und die Grundsätze der Auszahlung dieser Leistungen (GBl. der Republik Polen Nr. 10, Pos. 49).

$19 \S 21$ Abs. 5 der Verordnung über das Vorgehen bei Pensions- und Rentenleistungen und die Grundsätze der Auszahlung dieser Leistungen (GBl. der Republik Polen Nr. 10, Pos. 49).

$20 \mathrm{Im}$ Einzelnen Pogonowski, Postępowanie dowodowe $\mathrm{w}$ sprawach $\mathrm{z}$ zakresu ubezpieczeń społecznych prowadzone przez Zakład Ubezpieczeń Społecznych (Vorgehen beim Nachweis von Sozialversicherung durch die Sozialversicherungsanstalt), IPiSS (Institut der Arbeit und der Sozialfragen), Warschau 2006, S. 80. 
$\mathrm{Ab}$ dem 1. Januar 2009 ist die Einstellung von Arbeitnehmern für Tätigkeiten unter besonderen Bedingungen oder mit besonderem Charakter mit der Verpflichtung des Arbeitgebers verbunden, den vollen Versicherungsbeitrag an den Fonds für Überbrückungsaltersversorgung (FEP) in einer Höhe von 1,5\% der Grundveranlagung einzuzahlen. Aus diesem Fonds werden die Überbrückungsrenten finanziert. Organisatorische Barrieren bei der Benachrichtigung der ZUS über die Beschäftigungszeiträume unter besonderen Bedingungen oder mit besonderem Charakter können darin bestehen, dass dem Rentenorgan die Angaben über eine solche Beschäftigung nicht gemeldet werden oder eine solche Beschäftigung vom Arbeitgeber falsch eingestuft wird oder auch, dass der Arbeitgeber die Finanzierung von Beiträgen an den FEP bewusst zur Einsparung von Kosten unterlässt. Aus diesen Gründen schließt das Gesetz die Möglichkeit, dass der Arbeitnehmer mittels anderer Beweise den Nachweis über den Beschäftigungszeitraum erbringt, nicht aus. ${ }^{21}$

\section{Die Berücksichtigung von Auslandstätigkeiten}

Angesichts dessen stellt sich die Frage, inwieweit eine Berücksichtigung des Beschäftigungszeitraumes eines Arbeitnehmers unter besonderen Bedingungen oder mit besonderem Charakter bei einem ausländischen Arbeitgeber zulässig ist.

Im polnischen Sozialversicherungsrecht gilt seit vielen Jahren der Grundsatz, dass als Beitragszeitraum ausschließlich der Beschäftigungszeitraum polnischer Bürger im Ausland anerkannt wird, für den Sozialversicherungsbeiträge in Polen gezahlt wurden. Ausnahmen können im Gesetz vorgesehen oder in ratifizierten Auslandsverträgen enthalten sein 22 . Dieser Grundsatz fand auch bei der Berechtigung zur Zuerkennung einer vorzeitigen Überbrückungsrente im Hinblick auf Beschäftigungszeiträume unter besonderen Bedingungen oder mit besonderem Charakter bei ausländischen Arbeitnehmern Berücksichtigung. So wurde angenommen, dass auch die Beschäftigungszeit im Ausland bei ausländischen Arbeitgebern zu den nachgewiesenen Beschäftigungszeiträumen zu zählen ist. Dies betrifft Tätigkeiten, die im Katalog der Anlage zur Verordnung vom 7. Februar 1983 enthalten und als Beitragszeitraum anzuerkennen sind. ${ }^{23}$ Diese Ansicht vertritt auch das Oberste Gericht in seinem Urteil vom 27. April 201024, in dem festge-

21 Zieleniecki, Emerytura pomostowa w nowym systemie emerytalnym (Fußn. 13), S. 232.

22 Art. 6 Abs. 2 Pkt. 1d, Art. 6 Abs. 2 Pkt. 1b und c sowie Art. 8 des Gesetzes über Pension und Rentenversorgung aus dem FUS. Vgl. auch Urteil des Obersten Gerichts vom 30. August 2001, II UKN 500/00, OSNP 2003/10/258.

23 Urteile des Obersten Gerichts vom 5. März 2003, II UK196/02, OSNP 2004/8/144 und vom 5. November 2009, II UK 108/09, unveröffentlicht.

24 Urteil des Obersten Gerichts vom 27. April 2010, II UK 328/09, LEX Nr. 590252. Vgl. auch Maniewska, Praca w szczególnych warunkach wykonywana za granicą u pracodawcy zagranicznego 
stellt wird, dass Beschäftigungszeiträume unter besonderen Bedingungen oder mit besonderem Charakter im Ausland auf der Grundlage von direkt mit dem ausländischen Arbeitgeber geschlossenen Arbeitsverträgen als Beschäftigungszeiträume im Sinne von Art. 32 Abs. 1, 2, und 4 des Gesetzes über Altersversorgung aus dem FUS anzuerkennen sind. Doch dies gilt nur unter der Bedingung, dass auf der Grundlage von Arbeitsverträgen oder von internationalen Verträgen nach den im Beschäftigungsland geltenden Vorschriften in Polen ebenfalls eine Beitragszahlung in eine freiwillige Altersvorsorge- und Rentenversicherung erfolgt ${ }^{25}$ (z. B. Versicherungspflicht für Arbeitnehmer durch den ausländischen Arbeitgeber an dessen Sitz im Ausland oder an einem anderen Ort seiner Geschäftstätigkeit). In diesem Fall können die Beschäftigungszeiträume unter besonderen Bedingungen oder mit besonderem Charakter bei einem ausländischen Arbeitgeber im Ausland bei der Zuerkennung des Rechts auf vorzeitige Altersversorgung ohne Berücksichtigung der in Polen in eine freiwillige Rentenversicherung eingezahlten Beiträge anerkannt werden, wenn dies ein durch Polen ratifizierter internationaler Vertrag vorsieht 26 .

Die Grundsätze der Anrechnung von Beschäftigungszeiträumen für Tätigkeiten unter besonderen Bedingungen oder mit besonderem Charakter auf dem Gebiet eines anderen EU-Staates als Polen sind in der Verordnung Nr. 883/2004 des Europäischen Parlaments und des Rates vom 29. April 2004 zur Koordinierung der Systeme der sozialen Sicherheit ${ }^{27}$ festgelegt. Für die Zusammenrechnung der Zeiträume gilt Art. 6 der Verordnung 883/2004: Der zuständige Träger eines Mitgliedstaats, dessen Rechtsvorschriften den Erwerb, die Aufrechterhaltung, die Dauer oder das Wiederaufleben des Leistungsanspruchs, die Anwendung bestimmter Rechtsvorschriften oder den Zugang zu bzw. die Befreiung von der Pflichtversicherung, der freiwilligen Versicherung oder der freiwilligen Weiterversicherung von der Zurücklegung von Versicherungszeiten, Beschäftigungszeiten, Zeiten einer selbstständigen Erwerbstätigkeit oder Wohnzeiten abhängig machen, berücksichtigt soweit erforderlich die nach den Rechtsvorschriften eines anderen Mitgliedstaats zurückgelegten Versicherungszeiten, Beschäftigungszeiten, Zeiten einer selbstständigen Erwerbstätigkeit oder Wohnzeiten, als ob es sich um Zeiten handeln würde, die nach den für diesen Träger geltenden Rechtsvorschriften zurückgelegt worden sind.

Das Prinzip der Zusammenrechnung wurde erweitert in Art. 51 Abs. 1 der Verordnung Nr. 883/2004: Ist nach den Rechtsvorschriften eines Mitgliedstaats die Gewährung bestimmter Leistungen davon abhängig, dass die Versicherungszeiten nur in einer be-

(im Ausland bei ausländischen Arbeitgebern ausgeführte Tätigkeiten unter besonderen Bedingungen), Praca i Zabezpieczenie Społeczne (Arbeit und Soziale Sicherung) Nr. 9 (2010), S. 34-35.

25 Art. 6 Abs. 1 und 2 des Gesetzes über Altersversorgung und Renten aus dem FUS mit Art. 7 Pkt. 3 des Gesetzes vom 13. Oktober 1998 über das Sozialversicherungssystem (GB1. der Republik Polen Nr. 205, Pos. 1585, 2009).

26 Zieleniecki, Emerytura pomostowa w nowym systemie emerytalnym (Fußn. 13), S. 221.

27 ABl. der EU L 166/1. 
stimmten Beschäftigung oder selbstständigen Erwerbstätigkeit oder einem Beruf zurückgelegt wurden, für die ein Sondersystem für beschäftigte oder selbstständig erwerbstätige Personen gilt, so berücksichtigt der zuständige Träger dieses Mitgliedstaats die nach den Rechtsvorschriften eines anderen Mitgliedstaats zurückgelegten Zeiten nur dann, wenn sie in einem entsprechenden System, oder, falls es ein solches nicht gibt, in dem gleichen Beruf oder gegebenenfalls in der gleichen Beschäftigung oder selbstständigen Erwerbstätigkeit zurückgelegt wurden ${ }^{28}$. Falls der Interessent bei Berücksichtigung so erfüllter Zeiträume die Bedingungen zum Erhalt von Leistungen aus einem besonderen System nicht erfüllt, sind diese Zeiträume zum Erhalt von Leistungen im Rahmen des allgemeinen Systems berücksichtigt. Wenn auch dies nicht zutrifft, dann erfolgt die Berücksichtigung im Rahmen des betreffenden Systems für körperliche oder geistige Arbeit verrichtende Arbeitnehmer, und zwar unter der Bedingung, dass der Interessent einem dieser Systeme beitritt.

Die Art der Realisierung des Zusammenrechnungsprinzips von Zeiträumen ist festgelegt in Art. 12 der Verordnung Nr. 987/2009 des Europäischen Parlaments und Rates vom 16. September 2009 zur Festlegung der Modalitäten für die Durchführung der Verordnung (EG) Nr. 883/2004 über die Koordinierung der Systeme der sozialen Sicherheit ${ }^{29}$. Nach Art. 12 Abs. 1 und 2 dieser Verordnung setzen sich die betreffenden Institutionen der Mitgliedsländer in Verbindung, deren Gesetzgebung der Interessent ebenfalls unterstanden hat, um alle Beschäftigungszeiträume zur Anwendung des Zusammenrechnungsprinzips festzustellen. Entsprechende Versicherungszeiträume, die sich entsprechend der Regeln des betreffenden Mitgliedslands aus Lohnarbeit, selbstständiger Erwerbstätigkeit oder dem Wohnort ergeben, werden zusammengerechnet mit den entsprechend der jeweiligen Gesetzgebung in einem anderen Mitgliedsland erfüllten Zeiträumen, soweit dies notwendig ist zur Anwendung des Zusammenrechnungsprinzips und die Zeiträume sich nicht überdecken.

Die Frage der Berücksichtigung von Beschäftigungszeiträumen unter besonderen Bedingungen oder mit besonderem Charakter sowie von Tätigkeiten unter besonderen Bedingungen oder mit besonderem Charakter im Sinne von Art. 3 Abs. 1 und 3 des Gesetzes über Überbrückungsrenten bei der Feststellung des Rechts auf Überbrückungsrente kann jedoch Zweifel hervorrufen, wenn diese Zeiträume nach dem 31. Dezember 2008 bei ausländischen Arbeitgebern erzielt wurden. Ab dem 1. Januar 2009 bewirkt die Einstellung von Arbeitnehmern für solche Tätigkeiten auf der Seite der Beitragszahler und der ZUS organisatorisch-finanziellen Obliegenheiten: 1. die Führung von Verzeichnissen von Arbeitsplätzen, an denen solche Arbeiten ausgeführt werden, 2. die Führung von Verzeichnissen von den Arbeitnehmern, die solche Tätigkeiten ausführen, 3. die Übermittlung von Angaben an die ZUS zur Anzahl der Arbeitsplätze unter besonderen Bedingungen und mit besonderem Charakter, 4. die Übermittlung von Angaben an die ZUS zu den in den Verzeichnissen erfassten Arbeitnehmern, 5. die Führung

28 Zieleniecki, Emerytura pomostowa w nowym systemie emerytalnym (Fußn. 13), S. 222.

29 ABl. der EU L 284/1. 
eines Zentralregisters von Arbeitsplätzen, an denen Tätigkeiten unter besonderen Bedingungen und mit besonderem Charakter ausgeführt werden, 6. die Führung eines Zentralregisters über die Arbeitnehmer, die solche Tätigkeiten ausführen, 7. die $\mathrm{Zu}$ sammenstellung von Angaben über den Beschäftigungszeitraum unter besonderen Bedingungen oder mit besonderem Charakter auf dem individuellen Konto des Versicherten bei der ZUS, 8. die Zahlung von Beiträgen an den FEP, 9. die Registrierung von Angaben über die Anzahl der Arbeitnehmer, für die der Beitrag an den FEP gezahlt wird, sowie über die Beiträge an diesen Fonds durch die ZUS. Diese Obliegenheiten betreffen jedoch nicht ausländische Arbeitgeber, die weder ihren Sitz noch eine Vertretung in Polen haben und die polnische Bürger im Ausland oder auf dem Gebiet der Polnischen Republik zu Tätigkeiten unter besonderen Bedingungen oder mit besonderem Charakter beschäftigen. Solch eine Beschäftigung zieht nämlich keine Versicherungspflicht im polnischen Versicherungssystem nach sich. ${ }^{30}$

Das Urteil des Obersten Gerichts vom 27. April 201031, in dem die Berücksichtigung von Zeiträumen einer Tätigkeit unter besonderen Bedingungen oder mit besonderem Charakter zu Gunsten eines ausländischen Arbeitgebers bei der Zuerkennung des Rechts auf vorzeitige Altersversorgung grundsätzlich abhängig gemacht wird von der Beitragszahlung für eine freiwillige Pensionsrentenversicherung durch den Versicherten in Polen, findet darüber hinaus auch Anwendung bei der Feststellung des Beschäftigungszeitraumes hinsichtlich der qualifizierten Tätigkeit, von der die Berechtigung auf Überbrückungsrente abhängig ist. Nach Art. 2 Pkt. 2 des Gesetzes über das Sozialversicherungssystem ist der Beitragszahler nicht nur der Arbeitgeber im Sinne von Art. 4 Pkt. 2a des Gesetzes über das Sozialversicherungssystem, sondern auch der Versicherte ist zur Zahlung von Beiträgen für seine Sozialversicherung verpflichtet. Bei Arbeitnehmern, die Tätigkeiten unter besonderen Bedingungen sowie mit besonderem Charakter im Sinne von Art. 3 Abs. 1 und 3 des Gesetzes über Überbrückungsrenten zu Gunsten ausländischer Arbeitgeber ausführen, ruht die Verpflichtung zur Meldung einer Ausführung der betreffenden Tätigkeit an die ZUS sowie die Beitragszahlung an den FEP damit auf dem Versicherten selbst ${ }^{32}$.

Die Erfüllung der Bedingung einer Beitragszahlung für eine freiwillige Rentenversicherung sowie an den FEP wird nicht von Arbeitnehmern verlangt, die für Tätigkeiten unter besonderen Bedingungen oder mit besonderem Charakter im Sinne von Art. 3 Abs. 1 und 3 des Gesetzes über Überbrückungsrenten bei einem ausländischen Arbeitgeber angestellt sind und einer Rentenversicherung im für den Ausführungsort der Tätigkeit zuständigen Rentenversicherungssystem unterliegen, falls das Gesetz oder ein internationaler Vertrag die Obliegenheit enthält, diese Zeiträume bei der Zuerkennung der Berechtigung auf Leistungen auf Grundlage der Vorschriften des polnischen Rechts zu berücksichtigen. In diesem Fall ist jedoch der Nachweis der Zeiträume solch einer

30 Zieleniecki, Emerytura pomostowa w nowym systemie emerytalnym (Fußn. 13), S. 236-237.

31 Urteil des Obersten Gerichts vom 27. April 2010, II UK 328/09, LEX Nr. 590252.

32 Zieleniecki, Emerytura pomostowa w nowym systemie emerytalnym (Fußn. 13), S. 236-237. 
Beschäftigung mittels anderer Beweise als der auf dem individuellen Konto des Versicherten bei der ZUS sowie der im Zentralregister registrierten Angaben erforderlich. Ein ähnliches Problem betrifft auch solche Arbeitnehmer, die ihre Beschäftigungszeiträume durch qualifizierte Tätigkeiten in einem anderen EU- oder EFTA-Land als Polen erzielt haben und die sich um deren Anerkennung bei der Zuerkennung des Rechts auf Überbrückungsrente auf Grundlage der Vorschriften der Verordnung 883/2004 bemühen. ${ }^{33}$

Die Beweiserbringung für den Nachweis von Beschäftigungszeiträumen in Tätigkeiten unter besonderen Bedingungen oder mit besonderem Charakter, die in einem anderen EU-Mitgliedsland als Polen erbracht wurden, ist in den Vorschriften Kapitel II, Titel I der Verordnung 987/2009 geregelt. Im Einklang mit Art. 3 Abs. 2 dieser Verordnung sind die Personen, auf die die Verordnung 883/2004 Anwendung findet, verpflichtet, der zuständigen Institution Angaben, Unterlagen oder Beweise für die Bestätigung ihrer Lage oder die Lage ihrer Familie zur Zuerkennung oder Beibehaltung ihrer Rechte und Pflichten sowie auch zur Bestimmung der anzuwendenden Gesetze und der daraus folgenden Obliegenheiten dieser Personen zugänglich zu machen. Die von einer Institution eines Mitgliedslands unter Anwendung der Verordnungen 883/2004 und 987/2009 ausgestellten Unterlagen, die eine Bestätigung der Lage der betreffenden Person darstellen, werden von den Einrichtungen der übrigen Mitgliedsländer so lange anerkannt, so lange sie nicht eingezogen oder durch die sie ausstellenden Mitgliedsländer für ungültig erklärt werden. Bei Zweifeln hinsichtlich der Gültigkeit der Unterlagen oder der Richtigkeit der dargestellten Umstände, auf die sich die in den Unterlagen enthaltenen Angaben stützen, wendet sich die Empfängereinrichtung des Mitgliedslands an die die Unterlagen ausstellende Institution mit der Bitte um Klärung sowie auch gegebenenfalls um Einziehung dieser Unterlagen. Die die Unterlagen ausstellende Einrichtung prüft dann erneut die Grundlagen für deren Ausfertigung und zieht die Unterlagen gegebenenfalls ein. ${ }^{34}$

\section{E. Die Etablierung eines Systems zum elektronischen Datenaustausch}

Die Verordnungen 883/2004 und 987/2009 bilden die Grundlage für die Schaffung eines Systems des elektronischen Datenaustausches im Bereich der Sozialversicherungen (Electronic Exchange of Social Security Information - EESSI). Art. 78 Abs. 1 der Verordnung 883/2004 verpflichtet die Mitgliedsländer zur schrittweisen Nutzung neuer Technologien, die den Austausch, den Zugang und die Verarbeitung von Daten ermöglichen bzw. erleichtern und daher erforderlich sind zur Anwendung der Vorschriften beider Verfügungen. Die Kommission unterstützt die Maßnahmen, die Gegenstand gemeinsamen Interesses sind, sobald die Mitgliedsländer solche Datenverarbeitungstechniken eingeführt haben. Die Regierungen oder Institutionen der Mitgliedsländer dürfen

33 Ebd., S. 237.

34 Art. 5 Abs. 1 und 2 der Verordnung 987/2009. 
von den Institutionen eines anderen Mitgliedsstaates gesendete oder ausgestellte elektronische Unterlagen entsprechend der Vorschriften beider Verordnungen nicht deshalb ablehnen, weil sie auf elektronischem Weg erhalten wurden, wenn die empfangende Einrichtung zuvor erklärt hatte, dass sie elektronische Unterlagen empfangen kann. Kopien und gespeicherte Versionen solcher Unterlagen sind als gültig und als genaue Kopie des Originals oder Widerspiegelung der Angaben, auf die sich die Unterlage bezieht, zu betrachten, falls keine Gegenbeweise vorliegen. ${ }^{35}$

Der Austausch von Daten zwischen den Regierungen und staatlichen Institutionen der Mitgliedsländer und den von der Verordnung 883/2004 erfassten Personen stützt sich auf die Grundsätze öffentlicher Leistung, Effektivität, aktiver Unterstützung, schneller Zustellung und Zugänglichkeit, und dies eben auch auf elektronischem Wege, insbesondere für behinderte oder ältere Personen. Die Institutionen übermitteln oder tauschen unverzüglich untereinander alle Angaben aus, die notwendig sind zur Bestimmung der Rechte und Pflichten von Personen, die die Verordnung 883/2004 betrifft. Die Übermittlung dieser Daten und Angaben zwischen den Mitgliedsländern erfolgt direkt zwischen den Institutionen oder über Telekommunikationseinrichtungen ${ }^{36}$.

35 Art. 2 Abs. 1 und 2 der Verordnung 987/2009.

36 Ebd. 


\title{
Praktische Fragen der Inanspruchnahme von Freizügigkeit*
}

\author{
Stephan Fasshauer
}

A. Vorbemerkungen 235

B. Anmerkungen zur quantitativen Bedeutung der Fragestellung 237

C. Anmerkungen zu den Rechtsanwendungen 239

D. Zentrale Fragestellungen aus Sicht der Praxis 242

I. Fragen im Rahmen der Erwerbsphase $\quad 242$

II. Fragen im Rahmen des Rentenbezugs 246

1. Vorbemerkungen 246

$\begin{array}{ll}\text { 2. Berechtigung auf Leistungen nach dem deutsch-polnischen } & \\ \text { Sozialversicherungsabkommen von } 1975 \text { (DPSVA 1975) } & 247\end{array}$

3. Berechtigung auf Leistungen nach dem Fremdrentengesetz (FRG) 248

4. Weitere Entwicklung $\quad 249$

III. Beitreibung von Forderungen 250

IV. Elektronischer Datenaustausch 251

1. Überblick 251

2. Electronic Exchange of Social Security Information (EESSI) 252

3. Europäisches Online-Auskunftsverfahren mit Polen (EOA-Polen) 253

4. Sterbedatenabgleich Deutschland-Polen 254

E. Fazit 255

\section{A. Vorbemerkungen}

Seit Gründung der Europäischen Union bzw. Europäischen Gemeinschaft vor mehr als fünf Jahrzehnten erlebte diese in verschiedenen Etappen eine erhebliche Erweiterung. Nunmehr bilden 27 Staaten mit fast einer halben Milliarde Menschen die Europäische Union. Alle Länder vereint das Ziel, einen gemeinsamen europäischen Binnenmarkt zu bilden und damit die Voraussetzungen dafür zu schaffen, dass für Personen, Waren, Dienstleistungen und Kapital dieselbe Freizügigkeit zwischen den Mitgliedstaaten besteht wie innerhalb der einzelnen Länder. Das bedeutet, dass nicht nur grenzüber-

* Der Verfasser dankt Frau Seidel und Herrn Scheewe von der Deutschen Rentenversicherung BerlinBrandenburg für ihre wertvolle Unterstützung bei der Erstellung des Manuskriptes. 